\title{
MAPPING THEOREMS ON MESOCOMPACT SPACES
}

\author{
KUO-SHIH KAO AND LI-SHENG WU
}

\begin{abstract}
In this paper we prove two mapping theorems on mesocompact spaces: (1) Perfect mappings preserve mesocompactness; (2) Closed mappings preserve normal mesocompactness.
\end{abstract}

The main results of this paper are two mapping theorems on mesocompact spaces. Mesocompactness was defined in J. R. Boone [4] and studied by V. J. Mancuso [10] and J. R. Boone $[4,5]$. Mancuso [10] intended to prove that perfect mappings preserve mesocompactness, but his proof was incorrect. J. R. Boone [5] noticed the error in Mancuso's proof but he gave a proof only for a special case (the domains of the mappings were assumed to be normal). Our Theorem 1 solves the Mancuso problem. Boone [6] studied $k$-quotient mappings and proved that $k$-quotient, closed mappings preserve normal mesocompactness. Our Theorem 2 improves the foregoing result by omitting the condition " $k$-quotient" in the statement.

In this paper, normal spaces are assumed to be $T_{1}$, and all mappings are continuous and surjective. Let $\mathcal{Q}$ be a collection of subsets of $X$, the union $\cup\{U: U \in \mathcal{Q}\}$ is denoted by $\mathcal{U}^{*}$. For any $B \subset X$, let $(\mathcal{Q})_{B}=\{U \in \mathscr{Q}: U \cap B \neq$ $\varnothing\}$ and $(\mathscr{Q})_{\{x\}}$ is replaced by $(\mathscr{U})_{x}$. For the meanings of concepts used without definition in this paper, see [8 and 9].

Definition 1. A collection $\mathcal{Q}$ of subsets of $X$ is called compact-finite, if for each compact subset $K \subset X,(\mathscr{U})_{K}$ is finite.

DEFINITION 2 [4]. A topological space $X$ is called mesocompact if every open cover of the space has a compact-finite open refinement.

It is well known that

$$
\text { paracompact } \rightarrow \text { mesocompact } \rightarrow \text { metacompact }
$$

and none of the implications can be reversed.

Definition 3. Let $\mathcal{U}$ and $\mathcal{V}$ be two collections of subsets of $X$, we say that $\mathscr{U}$ is a compactwise $W$-refinement of $\mathfrak{V}$, if $\mathscr{Q}^{*}=\mathfrak{V}^{*}$ and for each compact subset $K \subset X$, the collection $(\mathscr{U})_{K}$ is a partial refinement of some finite subcollection $\mathcal{V}^{\prime}$ of $\mathscr{V}$.

In the proofs of the following lemmas and Proposition 1, we use the techniques invented by Junnila [8 and 9].

Received by the editors September 13, 1982 and, in revised form, January 19, 1983.

1980 Mathematics Subject Classification. Primary 54D20, 54C10.

Key words and phrases. Paracompact, metacompact, mesocompact, isocompact, semiopen cover, directed cover, compact-covering mapping, pseudo-open mapping.

(C)1983 American Mathematical Society $0002-9939 / 83 \$ 1.00+\$ .25$ per page 
LEMMA 1. Let $\left\{\mathcal{U}_{n}\right\}_{n \in N}$ be a sequence of open covers of $X$ such that for each $n \in N$, $\mathcal{Q}_{n+1}$ is a compactwise $W$-refinement of $\mathcal{U}_{n}$. Then $\mathcal{Q}_{1}$ has an open refinement $\mathcal{T}^{\prime}=\cup_{n=2}^{\infty} \mathcal{V}_{n}$ such that each $\mathcal{V}_{n}$ is a compact-finite collection.

Proof. Because each $\mathscr{U}_{n+1}$ is also a pointwise $W$-refinement of $\mathscr{Q}_{n}$, by $[8$, Proposition 2.2], $\mathcal{U}_{1}$ has an open refinement $\mathcal{T}=\cup_{n=2}^{\infty} \mathcal{V}_{n}$, such that for each $n \in N$ and $B \subset X$, if $\left(\mathcal{U}_{n+1}\right)_{B}$ is a partial refinement of a subcollection $\mathcal{U}^{\prime}$ of $\mathcal{Q}_{n}$, then $\left|\left(\mathscr{V}_{n+1}\right)_{B}\right| \leqslant\left|\mathcal{U}^{\prime}\right|$. Now for each compact subset $K \subset X$, there exists a finite subcollection $\mathcal{U}^{\prime}$ of $\mathscr{U}_{n}$ such that $\left(\mathcal{U}_{n+1}\right)_{K}$ is a partial refinement of $\mathcal{U}^{\prime}$, so $\left|\left(\mathscr{V}_{n+1}\right)_{K}\right| \leqslant\left|\mathcal{Q}^{\prime}\right|<\infty$, that is, $\mathscr{V}_{n}$ is compact-finite for each $n \geqslant 2$.

LEMMA 2. If an open cover of a topological space has a compact-finite semiopen refinement, then the cover has an open compactwise $W$-refinement.

Proof. The proof is similar to the proof of $[9$, Lemma 1.2].

Proposition 1. The following conditions are mutually equivalent for a topological space:

(1) The space is mesocompact.

(2) Every open cover of the space has a compact-finite semiopen refinement.

(3) Every open cover of the space has an open compactwise $W$-refinement.

(4) Every directed open cover of the space has a closure-preserving closed refinement which is refined by the collection consisting of all compact subsets.

Proof. (1) $\Rightarrow(2)$ is obvious. (2) $\Rightarrow$ (3) follows from Lemma 2.

$(3) \Rightarrow(1)$. By Lemma 1 , every open cover $\mathscr{U}$ of $X$ has an open refinernent $\mathscr{U} \int=\cup_{n=1}^{\infty} \mathcal{W}$, each $\mathcal{W}_{n}$ is compact-finite. For each $n \in N$, let $R_{n}=\cup_{k=1}^{n} \mathscr{Q} \int_{k}^{*}$, then $\Re=\left\{R_{n}: n \in N\right\}$ is a directed open cover of $X$. Let $\mathscr{P}$ be an open compactwise $W$-refinement of $\Re$. Let $F_{0}=\varnothing$ and for each $n \in N$, let $F_{n}=\{x \in X: \operatorname{St}(x, \mathscr{P}) \subset$ $\left.R_{n}\right\}$, note that each set $F_{n}$ is closed. For each $n \in N$, let $\mathcal{V}_{n}=\left\{W-F_{n-1}: W \in \mathcal{O} \int_{n}\right\}$, it is easily seen that $\mathscr{V}=\cup_{n=1}^{\infty} \mathcal{T}_{n}$ is an open refinement of $\mathscr{Q}[\mathbf{8}$, Theorem 3.1, proof (ii) $\Rightarrow$ (i)].

Let $V \subset X$ be any compact subset. Because $G R$ is directed, there exists an integer $n(K)$ such that $\operatorname{St}(K, \mathscr{P}) \subset R_{n(K)}$, that is $K \subset F_{n(K)}$. Then we have

$$
\left|(\mathscr{V})_{K}\right| \leqslant \sum_{n=1}^{\infty}\left|\left(\mathcal{V}_{n}\right)_{K}\right|=\sum_{n=1}^{n(K)}\left|\left(\mathscr{V}_{n}\right)_{K}\right| \leqslant \sum_{n=1}^{n(K)}\left|\left(\left.\mathcal{Q}\right|_{n}\right)_{K}\right|<\infty
$$

Therefore $\mathcal{Q}$ is compact-finite.

$(1) \Rightarrow(4)$. Let $\mathcal{Q}$ be any directed open cover of $X$, then $\mathcal{Q}$ has a compact-finite open refinement $\mathcal{V}$. For each $U \in \mathcal{Q}$, let $F(U)=\{x \in X: \operatorname{St}(x, \mathcal{V}) \subset U\}$, then $\mathscr{F}=\{F(U): U \in \mathcal{Q}\}$ is a closure-preserving closed refinement of $\mathscr{Q}[8$, Lemma 2.3, proof (ii) $\Rightarrow$ (i)]. Suppose $K \subset X$ is a compact subset. Since $\mathcal{U}$ is directed, there exists some $U \in \mathcal{Q}$, such that $\operatorname{St}(K, \mathcal{V}) \subset U$, that is $K \subset F(U)$. Therefore $\mathscr{F}$ is refined by the collection consisting of all compact subsets.

$(4) \Rightarrow(3)$. By [8, Theorem 3.1], $X$ is metacompact. Suppose $\mathscr{Q}$ is any open cover of $X$, then $\mathcal{Q}$ has a point-finite open refinement $\mathcal{V}$. Let $\tilde{\mathcal{V}}$ be the collection consisting of 
all finite unions of sets from $\widetilde{T}$. As a directed open cover of $X, \tilde{T}$ has a closurepreserving closed refinement $\mathscr{F}$ which is refined by the collection consisting of all compact subsets. For each $x \in X$, let $W(x)=\cap(\mathcal{V})_{x}-\cup\{F \in \mathscr{F}: x \notin F\}$. Q $S=$ $\{W(x): x \in X\}$ is an open cover of $X$. For each $F \in \mathscr{F}$, let $\mathcal{T}_{F}$ be the finite subcollection of $\mathcal{V}$ such that $F \subset \mathcal{V}_{F}^{*} \in \tilde{\mathcal{T}}$. Then $(\mathscr{W})_{F}$ is a partial refinement of $\mathcal{V}_{F}$ [8, Lemma 2.3, Proof (i) $\Rightarrow$ (iii)].

Now for each compact subset $K \subset X$, there exists an $F(K) \in \mathscr{F}$ such that $K \subset F(K)$, so $(\mathcal{W})_{K}$ is a partial refinement of a finite subcollection $\mathcal{V}_{F(K)}$ of $\mathcal{V}$, that is, $\mathscr{Q} \int$ is an open compactwise $W$-refinement of $\tau^{\top}$ as well as of $\mathcal{Q}$.

Definition 4 (Michael [12]). A mapping $f: X \rightarrow Y$ is called compact-covering if, whenever $K$ is a compact set in $Y$, there exists a compact set $C$ in $X$ such that $f(C)=K$.

Proposition 2. The image of a mesocompact space under a closed and compactcovering mapping is mesocompact.

Proof. Let $\mathcal{T}=\left\{V_{\beta}\right\}_{\beta \in B}$ be a directed open cover of $Y$, then $\mathcal{Q}=\left\{f^{-1}\left(V_{\beta}\right)\right\}_{\beta \in B}$ is a directed open cover of $X$. By Proposition 1(4), थ has a closure-preserving closed refinement $\mathscr{F}=\left\{F_{\alpha}\right\}_{\alpha \in A}$, which is refined by the collection consisting of all compact subsets of $X$. Since $f$ is closed and compact-covering, $\left\{f\left(F_{\alpha}\right)\right\}_{\alpha \in A}$ is a closurepreserving closed refinement of $\mathcal{T}$, and is refined by the collection consisting of all compact subsets of $Y$. By Proposition 1(4), $Y$ is mesocompact.

Since every perfect mapping is compact-covering (e.g. [13]), we obtain the following theorem.

THEOREM 1. The image of a mesocompact space under a perfect mapping is mesocompact.

Definition 5 (BACON [3]). A topological space is called isocompact if every countably compact closed subset of $X$ is compact.

E. Michael [11] proved that if $X$ is a paracompact space and $f: X \rightarrow Y$ a closed mapping, then $f$ is also a compact-covering mapping. In his proof, the paracompactness is used only for turning a countably compact closed subset of a normal space to a compact one. We have the following lemma.

LEMMA 3. A closed mapping from a normal isocompact space $X$ onto a space $Y$ is also a compact-covering mapping.

THEOREM 2. The image of a normal mesocompact space under a closed mapping is normal mesocompact.

Proof. Let $f$ be a closed mapping from a normal mesocompact space $X$ onto a space $Y$. It is well known that $Y$ is normal. Because mesocompact space is isocompact (Arens-Dugundji [1]), so by Lemma 3, $f$ is also a compact-covering mapping. By Proposition 2, $Y$ is mesocompact.

REMARK. Junnila [9] proved that the image of a paracompact space under a pseudo-open and compact mapping is metacompact in order to answer the question of Arhangel'skii [2] affirmatively. Junnila's proof for the paracompact case can easily be modified to cover the mesocompact case. On the other hand, the image of a 
metacompact space under an open and compact mapping is not necessarily metacompact (Chaber [7]).

\section{REFERENCES}

1. R. Arens and J. Dugundji, Remark on the concept of compactness, Portugal. Math. 9 (1950), 141-143.

2. A. V. Arhangel'skii, The intersection of topologies, and pseudo-open compact mappings, Soviet Math. Dokl. 17 (1976), 160-163.

3. P. Bacon, The compactness of countably compact spaces, Pacific J. Math. 32 (1970), 587-592.

4. J. R. Boone, Some characterizations of paracompactness in k-spaces, Fund. Math. 72 (1971), $145-155$.

5. 69-74. , A note on mesocompact and sequentially mesocompact spaces, Pacific J. Math. 44 (1973),

6.

7. J. Chaber, Metacompactness and the class MOBI, Fund. Math. 91 (1976), 211-217.

8. H. J. K. Junnila, Metacompactness, paracompactness and interior-preserving open covers, Trans. Amer. Math. Soc. 249 (1979), 373-375.

9. $244-248$.

10. V. J. Mancuso, Mesocompactness and related properties, Pacific J. Math. 33 (1970), 345-355.

11. E. Michael, $A$ note on closed maps and compact sets, Israel J. Math. 2 (1964), 173-176.

12., $\boldsymbol{\aleph}_{0}$-spaces, J. Math. Mech. 15 (1966), 983-1002.

13. F. Siwiec and V. J. Mancuso, Relations among certain mappings and conditions for their equivalence, Topology Appl. 1 (1971), 33-41.

Department of Mathematics, Kiangsu Teachers' College, Suchow, Kiangsu, China 